\title{
THE PARAMETERS OF A CHAIN SEQUENCE
}

\author{
T. S. CHIHARA
}

(Communicated by J. Marshall Ash)

\begin{abstract}
We give a method for constructing explicitly all parameter sequences for any chain sequence for which one parameter sequence is known. An application to orthogonal polynomials associated with birth and death processes is given.
\end{abstract}

\section{INTRODUCTION}

A sequence $\left\{a_{n}\right\}_{n=1}^{\infty}$ is a (positive) chain sequence if there exists a second sequence $\left\{g_{n}\right\}_{n=0}^{\infty}$ such that

$$
\text { (i) } 0 \leq g_{0}<1, \quad 0<g_{n}<1 \quad \text { for } n \geq 1 \text {; }
$$

(ii) $a_{n}=\left(1-g_{n-1}\right) g_{n} \quad$ for $n \geq 1$.

Chain sequences seem to have appeared first in certain continued fractions studied by E. B. Van Vleck and the theory was formalized by Wall (see [13] for this and references). See [7,9] for some examples of more recent applications to continued fraction theory. Our interest in chain sequences stems from the useful role they play in the study of orthogonal polynomials and their zeros (see for example [1, 2, 3, 5, 6, 12]). This connection is not surprising in view of the close relation of orthogonal polynomials to continued fractions. In their original form, chain sequences were not restricted to be positive and for the more general case the above definition must be modified by changing the inequalities to weak inequalities. For the applications to orthogonal polynomials, only positive chain sequences are considered so we adopt the more restrictive definition above and will not use the adjective "positive".

The numbers $g_{n}$ above are called parameters of the chain sequence and $\left\{g_{n}\right\}$ is a parameter sequence for $\left\{a_{n}\right\}$. Every chain sequence has a minimal parameter sequence $\left\{m_{n}\right\}$ uniquely determined by the condition $m_{0}=0$, and it has a maximal parameter sequence $\left\{M_{n}\right\}$ which is characterised by the condition

Received by the editors February 2, 1989 and, in revised form, June 7, 1989. Presented to the AMS in Phoenix, Arizona on January 14, 1989.

1980 Mathematics Subject Classification. Primary 40A15; Secondary 42C05.

Key words and phrases. Chain sequence, continued fractions, orthogonal polynomials, birth and death process. 
$[13$, p. 82]

$$
\sum_{n=1}^{\infty} \frac{M_{1} M_{2} \cdots M_{n}}{\left(1-M_{1}\right)\left(1-M_{2}\right) \cdots\left(1-M_{n}\right)}=\infty .
$$

If $M_{0}>0$, then for every $g_{0}, 0<g_{0}<M_{0}$, there is a unique parameter sequence $\left\{g_{n}\right\}$ such that

$$
m_{n}<g_{n}<M_{n}, \quad n \geq 0 .
$$

It is frequently useful to know explicitly more than one parameter sequence for a given chain sequence but it is rare that more than one or two are explicitly known. In some instances, a non-minimal parameter sequence is known but the minimal parameter sequence is what is needed. In this paper, we will show how all parameter sequences can be found for any chain sequence for which one parameter sequence is explicitly known.

\section{NON-MINIMAL PARAMETERS KNOWN}

We first introduce the following notation: Let $g=\left\{g_{n}\right\}$ be any parameter sequence and define $P_{n}=P_{n}(g)$ by

$$
P_{0}(g)=1, \quad P_{n}(g)=\frac{g_{0} \cdots g_{n-1}}{\left(1-g_{0}\right) \cdots\left(1-g_{n-1}\right)}, \quad n \geq 1 .
$$

Now suppose that (1.1) holds with $g_{0}>0$. Define the sequence $\left\{S_{n}\right\}$ and the extended real number $S$ by

$$
S_{-1}=0, \quad S_{n}=\sum_{k=0}^{n} P_{k} \quad(n \geq 0), \quad S=\sum_{k=0}^{\infty} P_{k} .
$$

Next define $h_{n}=h_{n}(t)$ by

$$
h_{n}(t)=\frac{1+t S_{n-1}}{1+t S_{n}} g_{n}, \quad n \geq 0 .
$$

Since $S_{n}-g_{n} S_{n-1}=\left(1-g_{n}\right) S_{n+1}$, we have

$$
1-h_{n}(t)=\frac{1+t S_{n+1}}{1+t S_{n}}\left(1-g_{n}\right) \text {. }
$$

Thus $\left(1-h_{n-1}\right) h_{n}=\left(1-g_{n-1}\right) g_{n}=a_{n}(n \geq 1)$ and it is readily verified that $0 \leq h_{0}<1$ and $0<h_{n}<1 \quad(n \geq 1)$ if and only if

$$
-\frac{1}{S} \leq t \leq \infty
$$

(This includes the limiting case $S=\infty$.)

Thus with the restriction (2.5), (2.3) yields all parameter sequences $\left\{h_{n}\right\}$ for the chain sequence $\left\{a_{n}\right\}$. In particular, $h_{0}(\infty)=0$ so we get the minimal parameters for $t=\infty$. On the other hand, we have

$$
\begin{gathered}
\frac{h_{n}}{1-h_{n}}=\frac{1+t S_{n-1}}{1+t S_{n+1}} \cdot \frac{g_{n}}{1-g_{n}}, \\
P_{n}(h)=\frac{(1+t)\left(1+t S_{1}\right)}{\left(1+t S_{n}\right)\left(1+t S_{n+1}\right)} P_{n}(g) .
\end{gathered}
$$


Recalling the criterion (1.2), we see we will have the maximal parameters if $t=-1 / S$. This remains true if $S=\infty$ (in which case of course $h_{n}(0)=g_{n}$ is the $n$th maximal parameter).

Example. Take $a_{n}=a$ where $0<a \leq 1 / 4$. Then $a_{n}=(1-g) g$ with $g=[1+\sqrt{1-4 a}] / 2, n \geq 1$. Referring to (2.1)-(2.3), we have

$$
P_{n}=R^{n}, \quad R=\frac{1+\sqrt{1-4 a}}{1-\sqrt{1-4 a}}, \quad S_{n}=\frac{R^{n+1}-1}{R-1} .
$$

Hence all parameter sequences for $\{a\}$ are given by

$$
h_{n}(t)=\frac{1+\sqrt{1-4 a}}{2} \frac{R-1+\left(R^{n}-1\right) t}{R-1+\left(R^{n+1}-1\right) t}, \quad 0 \leq t \leq \infty .
$$

The corresponding maximal parameters $M_{n}=h_{n}(0)=g$ and the minimal parameters $m_{n}=h_{n}(\infty)$ were given by Wall [13, p. 83].

In the special case $a=1 / 4,(2.8)$ reduces neatly to

$$
h_{n}(t)=\frac{1+n t}{2[1+(n+1) t]} \text {. }
$$

\section{Minimal PARAMETERS ARE KNOWN}

Suppose next that

$$
a_{n}=\left(1-m_{n-1}\right) m_{n}, \quad n \geq 1,
$$

with $m_{0}=0,0<m_{n}<1 \quad(n \geq 1)$ and

$$
\sigma=\sum_{k=1}^{\infty} \frac{m_{1} \cdots m_{n}}{\left(1-m_{1}\right) \cdots\left(1-m_{n}\right)}<\infty .
$$

(If the series in (3.2) diverges, the minimal parameters are also the maximal parameters so the chain sequence has only the one parameter sequence.)

Now define a second sequence $\left\{b_{n}\right\}$ by

$$
b_{n}=a_{n+1}, \quad n \geq 1 \text {. }
$$

Then $b_{n}=\left(1-g_{n-1}\right) g_{n}$ where

$$
g_{n}=m_{n+1}, \quad n \geq 0,
$$

so $\left\{b_{n}\right\}$ is a chain sequence with the non-minimal parameter sequence $\left\{g_{n}\right\}$. Thus we can define $\left\{S_{n}\right\}$ and $S$ by (2.2). In particular, we have $S=1+\sigma$, where $\sigma$ is given by (3.2). For each $t$ satisfying (2.5), (2.3) defines a parameter sequence for $\left\{b_{n}\right\}$. As before, $h_{n}(-1 / S)\left(=M_{n+1}\right)$ is the $n$th maximal parameter for $\left\{b_{n}\right\}$ while $h_{n}(0)=g_{n}=m_{n+1}$. Also, we have

$$
a_{n+1}=b_{n}=\left(1-h_{n-1}\right) h_{n}, \quad n \geq 1 \text {, }
$$

where

$$
m_{n+1} \leq h_{n}(t) \leq M_{n+1} \quad \text { for }-\frac{1}{S} \leq t \leq 0
$$


Further, $h_{0}(t)=m_{1} /(1+t)$ so if we define $h_{-1}$ by

$$
h_{-1}=-t, \quad-\frac{1}{S} \leq t \leq 0,
$$

then

$$
\left(1-h_{-1}\right) h_{0}=m_{1}=a_{1} .
$$

Combined with (3.5), this shows that $\left\{h_{n}(t)\right\}_{n=-1}^{\infty}$ is a parameter sequence for $\left\{a_{n}\right\}_{n=1}^{\infty}$. Pulling all of this together, we set $r=-t, f_{n}(r)=h_{n-1}(t)$, and we can state in summary:

Let $\left\{a_{n}\right\}_{n=1}^{\infty}$ be a chain sequence with minimal parameter sequence $\left\{m_{n}\right\}_{n=0}^{\infty}$ satisfying (3.2). Let

$$
S_{-1}=0, \quad S_{0}=1, \quad S_{n}=1+\sum_{k=1}^{n} \frac{m_{1} \cdots m_{k}}{\left(1-m_{1}\right) \cdots\left(1-m_{k}\right)}, \quad n \geq 1
$$

Then all parameter sequences for $\left\{a_{n}\right\}$ are given by $\left\{f_{n}(r)\right\}$ where

$$
f_{0}(r)=r, f_{n}(r)=\frac{1-r S_{n-1}}{1-r S_{n}} m_{n}, \quad 0 \leq r \leq \frac{1}{S} .
$$

An example in which $S_{n}$ can be found in closed form is given by taking

$$
a_{n}=\frac{(2 n-1)(2 n+3)}{16 n(n+1)} \quad \text { with } m_{n}=\frac{n^{2}(2 n+3)}{4(n+1)^{3}} .
$$

All parameter sequences are then given by (3.8) with $0 \leq r \leq 3 / 4$ and $S_{n}=$ $(4 / 3)(n+1)(n+3)(n+2)^{-2}$.

\section{Applications}

If $\left\{f_{n}\right\}$ is any sequence, put

$$
\pi_{n}(f)=\frac{f_{1} f_{2} \cdots f_{n}}{\left(1-f_{1}\right)\left(1-f_{2}\right) \cdots\left(1-f_{n}\right)} .
$$

Then if $\left\{g_{n}\right\}$ and $\left\{h_{n}\right\}$ are any two non-maximal parameter sequences for the same chain sequence, $(2.6)$ shows that

$$
\lim _{n \rightarrow \infty} \frac{\pi_{n}(g)}{\pi_{n}(h)}=\frac{(1+t)\left(1+t S_{1}\right)}{(1+t S)^{2}}>0
$$

where $S$ (given by (2.2)) is finite and $t=\left(g_{0}-h_{0}\right) / h_{0}$. (We had previously known that $\lim _{n \rightarrow \infty} g_{n} / h_{n}=1$.)

Convergence of certain series involving $\pi_{n}(m)$, where $m$ is the minimal parameter sequence, figures prominently in the question of determinacy or indeterminacy of the Hamburger moment problems associated with orthogonal polynomials (see [5]). In many cases, we do not know the minimal parameter sequence but (4.2) shows that all non-maximal parameter sequences are asymptotically equivalent. 
As a second application, consider the problem of explicitly constructing a family of orthogonal polynomial sequences which are orthogonal over $[0, \infty)$ with respect to measures which differ from each other only by the size of the mass at the origin. This construction was described in [1, Th. 2] and is especially well suited to the case where the orthogonal polynomials are associated with a birth and death process. Specifically, let $\left\{Q_{n}(x)\right\}$ be defined by the recurrence formula (see [8])

$$
\begin{gathered}
-x Q_{n}(x)=\lambda_{n} Q_{n+1}(x)+\left(\lambda_{n}+\mu_{n}\right) Q_{n}(x)+\mu_{n} Q_{n-1}(x) \\
Q_{-1}(x)=0, \quad Q_{0}(x)=1, \quad \lambda_{i}>0, \quad \mu_{i+1}>0 \quad(i \geq 0), \quad \mu_{0} \geq 0 .
\end{gathered}
$$

These polynomials satisfy an orthogonality of the form

$$
\int_{0}^{\infty} Q_{m}(x) Q_{n}(x) d \psi(x)=\frac{\mu_{1} \cdots \mu_{n}}{\lambda_{0} \cdots \lambda_{n-1}} \delta_{m n} .
$$

Let us consider here the case where we have a reflecting barrier at 0 -that is, assume $\mu_{0}=0$. (If $\mu_{0}>0$, the procedure becomes a bit more complicated.)

We then consider the corresponding "dual process". That is, consider the process obtained from (4.3) after replacing $\lambda_{n}$ and $\mu_{n}$, respectively, by $\lambda_{n+1}$ and $\mu_{n+1}$. The resulting orthogonal polynomials are the "kernel polynomials" which are orthogonal with respect to the distribution $x d \psi(x)$. Consideration of the corresponding monic polynomials and the resulting recurrence relation leads to the chain sequence

$$
\frac{\lambda_{n} \mu_{n}}{\left(\lambda_{n-1}+\mu_{n}\right)\left(\lambda_{n}+\mu_{n+1}\right)}=\left(1-g_{n-1}\right) g_{n}, \quad g_{n}=\frac{\lambda_{n}}{\lambda_{n}+\mu_{n+1}} .
$$

Next obtain all other parameter sequences for (4.4). That is, put

$$
\rho_{n}(t)=\frac{\left(1+t S_{n-1}\right)}{1+t S_{n}}
$$

where $S_{n}$ is given by (2.2), and then define

$$
\lambda_{n}(t)=\rho_{n} \lambda_{n}, \quad \mu_{0}(t)=0, \quad \mu_{n+1}(t)=\frac{1}{\rho_{n}} \mu_{n+1} .
$$

Now consider the orthogonal polynomials defined by (4.3) after replacing $\lambda_{n}$ and $\mu_{n}$ by $\lambda_{n}(t)$ and $\mu_{n}(t)$, respectively. Then the following is true [1, Theorem 2]: for $t=-1 / S$ (corresponding to the maximal parameters of (4.4)), the corresponding polynomials will be orthogonal over $[0, \infty)$ with respect to a distribution $d \varphi(x)$ where $\varphi$ is the solution of a determined Hamburger moment problem and is continuous at the origin. For all other finite values of $t$ (corresponding to all other non-minimal parameter sequences), the corresponding polynomials will be orthogonal with respect to the distribution which is obtained from $d \varphi(x)$ by adding positive mass $J$ at the origin, the mass being given by the formula

$$
J=(1+t) \frac{S}{S-1}\left[\varphi(\infty)-\varphi\left(0^{-}\right)\right]
$$


Note that the moment problem associated with the original orthogonal polynomial sequence $\left\{Q_{n}(x)\right\}$ could be indeterminate. Thus if a polynomial sequence is orthogonal over $[0, \infty)$ with respect to the solution of an indeterminate moment problem, it is also orthogonal with respect to a distribution which is obtained by adding mass at the origin to a solution of a determined Hamburger moment problem.

The above is a more efficient and generally applicable method of determining this family of orthogonal polynomials than that used in [4]. For other approaches to the problem of determining orthogonal polynomials when masses are added to measures under various conditions, see [10, 11$]$.

\section{REFERENCES}

1. T. S. Chihara, Chain sequences and orthogonal polynomials, Trans. Amer. Math. Soc. 104 (1962), 1-16.

2. __ An introduction to orthogonal polynomials, Gordon and Breach, New York, 1978.

3. _ Spectral properties of orthogonal polynomials on unbounded sets, Trans. Amer. Math. Soc. 270 (1982), 623-639.

4. __ Orthogonal polynomials and measures with end point masses, Rocky Mountain J. Math. 15 (1985), 705-719.

5. ___ Hamburger moment problems and orthogonal polynomials, Trans. Amer. Math. Soc., (to appear).

6. M. E. H. Ismail, Monotonicity of zeros of orthogonal polynomials, Proc. Conf. on $q$-series, $q$-Series and Partitions (edited by D. Stanton), the IMA volumes in Mathematics and its Applications, 18, Springer-Verlag, New York, 1989, pp. 177-190.

7. L. Jacobsen and D. Masson, On the convergence of limit periodic continued fractions $K\left(a_{n} / 1\right)$, where $a_{n} \rightarrow-1 / 4$, part III, Constructive Approx., (to appear).

8. S. Karlin and J. McGregor, The differential equations of birth and death processes and the Stieltjes moment problem, Trans. Amer. Math. Soc. 85 (1957), 489-546.

9. E. P. Merkes, On truncation errors for continued fraction computations, SIAM J. Numer. Anal. 3 (1966), 486-496.

10. P. G. Nevai, Orthogonal polynomials, Memoirs of Amer. Math. Soc., no. 213, Providence, Rhode Island, 1979.

11. V. Ouvarov, On the connection between systems of polynomials orthogonal with respect to different distributions, Zh. Vych. Mat. i mat. Fiz. 9, 6 (1969), 1253-1262.

12. E. A. Van Doorn, Representations and bounds for zeros of orthogonal polynomials and eigenvalues of sign-symmetric tridiagonal matrices, J. Approx. Theory 51 (1987), 254-266.

13. H. S. Wall, Analytic theory of continued fractions, van Nostrand, New York, 1948.

Department of Mathematical Sciences, Purdue University Calumet, Hammond, INDIANA 46323 